\title{
A framework for medical diagnosis via fuzzy soft matrices
}

\author{
Yildiray Celik and Ilknur Celik \\ Department of Mathematics, Ordu University, Ordu, Turkey
}

Received: 20 August 2015, Revised: 31 December 2015, Accepted: 26 January 2016

Published online: 28 April 2016.

\begin{abstract}
In this paper we introduce matrice represantation of fuzzy soft sets. By using the notion of fuzzy soft matrices, we apply fuzzy soft set technology through the well known Sanchez's [8] approach for medical diagnosis. Also we exhibit the technique with a hypothetical case study.
\end{abstract}

Keywords: Fuzzy soft set, fuzzy soft matrices, Sanchez's approach.

\section{Introduction}

Many complicated problems in economics, engineering, social science, medical science and many other fields involve uncertain data. These problems which one come face to face with in life cannot be solved using classical mathematic methods. In classical mathematics, a mathematical model of an object is devised and the notion of the exact solution of this model is determined. Because of that the mathematical model is too complex, the exact solution cannot be found. There are several well-known theories to describe uncertainty. For instance fuzzy set theory [1], rough set theory [2] and other mathematical tools. But all of these theories have their inherit difficulties as pointed out by Molodtsov [3]. To overcome these difficulties, Molodtsov introduced the concept of soft set as a new mathematical tool for dealing with uncertainties that is free from the difficulties affecting existing methods. The theory of soft sets has rich potential for applications in several directions, few of which had been demonstrated by Molodtsov in his pioneer work [3]. At present, works on soft set theory are making progress rapidly. Maji et al. [4] initiated the concept of fuzzy soft sets with some properties regarding fuzzy soft union, intersection, complement of a fuzzy soft set, De Morgan's Law etc. Neog and Sut [5] have reintroduced the notion of fuzzy soft sets and redefined the complement of a fuzzy soft set accordingly. They have shown that the modified definition of complement of a fuzzy soft set meets all the requirements that complement of a set in classical sense really does. Applications of Fuzzy Soft Set Theory in many disciplines and real life situations have been studied by many researchers. De et.al. [6] have studied Sanchez's [7,8] method of medical diagnosis using intuitionistic fuzzy set. Saikia et.al. [9] have extended the method in [6] using intuitionistic fuzzy soft set theory. In [10], Chetia and Das have studied Sanchez's approach of medical diagnosis through IVFSS obtaining an improvement of the same presented in De et.al. [6]. Using the representation of interval valued fuzzy matrix, Meenakshi et.al. [11] have provided the techniques to study Sanchez's approach of medical diagnosis of Interval valued fuzzy matrices. In this paper, by using the notion of fuzzy soft matrices, we apply fuzzy soft set technology through the well known Sanchez's [8] approach for medical diagnosis and we exhibit the technique with a hypothetical case study. 


\section{Preliminaries}

In this section, we will give some known and useful definitions and notations regarding soft set, fuzzy soft set.The definitions and notions, in this part, may be found in references $[1,3,4,12]$.

Definition 1. [3] Let $U$ be an inital universe set and $E$ be a set of parameters. The power set of $U$ is denoted by $\mathscr{P}(U)$ and $A$ is a subset of $E$. A pair $(F, A)$ is called a soft set over $U$, where $F$ is a mapping given by $F: A \rightarrow \mathscr{P}(U)$.

Definition 2. [1] A fuzzy subset $\mu$ of $U$ is defined as a map from $U$ to $[0,1]$. The family of all fuzzy subsets of $U$ is denoted by $\mathscr{F}(U)$. Let $\mu, v \in \mathscr{F}(U)$ and $x \in U$. Then the union and intersection of $\mu$ and $v$ are defined following way:

$$
\begin{aligned}
& (\mu \vee v)(x)=\mu(x) \vee v(x) \\
& (\mu \wedge v)(x)=\mu(x) \wedge v(x)
\end{aligned}
$$

$\mu \leq v$ if and only if $\mu(x) \leq v(x)$ for all $x \in U$

Definition 3. [4] Let $U$ be a common universe, $E$ be a set of parameters and $A \subseteq E$. Then a pair $(\widetilde{F}, A)$ is called a fuzzy soft set over $U$, where $\widetilde{F}$ is a mapping given by $\widetilde{F}: A \rightarrow \mathscr{F}(U)$.

Definition 4. [4] For two fuzzy soft sets $(\widetilde{F}, A)$ and $(\widetilde{G}, B)$ over a common universe $U$, we say that $(\widetilde{F}, A)$ is a fuzzy soft subset of $(\widetilde{G}, B)$ if

(i) $A \subseteq B$

(ii) $F(a) \leq G(a)$ for all $a \in A$.

In this case, we write $(\widetilde{F}, A) \widetilde{\widetilde{C}}(\widetilde{G}, B)$.

Definition 5. [4] The complement of fuzzy soft set $(\widetilde{F}, A)$ denoted by $(\widetilde{F}, A)^{c}$, is defined by $(\widetilde{F}, A)^{c}=(\widetilde{F}, A)$, where $\widetilde{\sim}^{c}: A \rightarrow \mathscr{F}(U)$ is mapping given by $\stackrel{\sim}{F}^{c}=[\widetilde{F}(a)]^{c}$ for all $a \in A$.

Definition 6. [4]

(i) A fuzzy soft set $(\widetilde{F}, A)$ is said to be absolute fuzzy soft set over $U$ if $\widetilde{F}(a)=U$ for all $a \in A$.

(ii) A fuzzy soft set $(\widetilde{F}, A)$ is said to be null fuzzy soft set over $U$ if $\widetilde{F}(a)=0_{U}$ for all $a \in A$.

Definition 7. [12] Let $(\widetilde{F}, A)$ and $(\widetilde{G}, B)$ be two fuzzy soft sets over a common universe $U$. Then,

(1) The union of fuzzy soft sets $(\widetilde{F}, A)$ and $(\widetilde{G}, B)$ is defined as the fuzzy soft set $(\widetilde{H}, C)=(\widetilde{F}, A) \widetilde{\cup}(\widetilde{G}, B)$ over $U$, where $C=A \cup B$ and

$$
\widetilde{H}(c)=\left\{\begin{array}{lll}
F(c) & \text { if } & c \in A \backslash B \\
G(c) & \text { if } & c \in B \backslash A \\
F(c) \vee G(c) & \text { if } & c \in A \cap B
\end{array}\right.
$$

for all $c \in C$.

(2) The intersection of fuzzy soft sets $(\widetilde{F}, A)$ and $(\widetilde{G}, B)$ is defined as the fuzzy soft set $(\widetilde{H}, C)=(\widetilde{F}, A) \widetilde{\cap}(\widetilde{G}, B)$ over $U$, where $C=A \cap B$ and $\widetilde{H}(c)=\widetilde{F}(c) \wedge \widetilde{G}(c)$ for all $c \in C$.

(3) The $\wedge$-intersection of fuzzy soft sets $(\widetilde{F}, A)$ and $(\widetilde{G}, B)$ is defined as the fuzzy soft set $(\widetilde{H}, C)=(\widetilde{F}, A) \widetilde{\wedge}(\widetilde{G}, B)$ over $U$, where $C=A \times B$ and $\widetilde{H}(a, b)=\widetilde{F}(a) \wedge \widetilde{G}(b)$ for all $(a, b) \in A \times B$. 
(4) The $\vee$-union of fuzzy soft sets $(\widetilde{F}, A)$ and $(\widetilde{F}, B)$ is defined as the fuzzy soft set $(\widetilde{H}, C)=(\widetilde{F}, A) \widetilde{V}(\widetilde{G}, B)$ over $U$, where $C=A \times B$ and $\widetilde{H}(a, b)=\widetilde{F}(a) \vee \widetilde{G}(b)$ for all $(a, b) \in A \times B$.

Example 1. Let $U=\left\{h_{1}, h_{2}, h_{3}\right\}$ be the set of three houses under consideration and

$$
E=\left\{e_{1}(\text { costly }), e_{2}(\text { beautiful }), e_{3}(\text { greensurroundings })\right\}
$$

be the set of parameters. Consider two fuzzy soft sets $(\widetilde{F}, A)$ and $(\widetilde{G}, B)$, where $A=\left\{e_{1}, e_{2}\right\}$ and $B=\left\{e_{1}, e_{2}, e_{3}\right\}$ given by

$$
\begin{aligned}
(\widetilde{F}, A) & =\left\{\widetilde{F}\left(e_{1}\right)=\left\{\left(h_{1}, .7\right),\left(h_{2}, .5\right),\left(h_{3}, .3\right)\right\}, \widetilde{F}\left(e_{2}\right)=\left\{\left(h_{1}, .7\right),\left(h_{2}, .6\right),\left(h_{3}, .5\right)\right\}\right\} \\
(\widetilde{G}, B) & =\left\{\widetilde{G}\left(e_{1}\right)=\left\{\left(h_{1}, .7\right),\left(h_{2}, .5\right),\left(h_{3}, .3\right)\right\}, \widetilde{G}\left(e_{2}\right)=\left\{\left(h_{1}, .7\right),\left(h_{2}, .6\right),\left(h_{3}, .5\right)\right\}\right. \\
& \left.\widetilde{G}\left(e_{3}\right)=\left\{\left(h_{1}, .2\right),\left(h_{2}, .4\right),\left(h_{3}, .5\right)\right\}\right\}
\end{aligned}
$$

Then,

(i) $(\widetilde{F}, A)^{c}=\left[\widetilde{F}\left(e_{1}\right)\right]^{c}=\left\{\left(h_{1}, .3\right),\left(h_{2}, .5\right),\left(h_{3}, .7\right)\right\},\left[\widetilde{F}\left(e_{2}\right)\right]^{c}=\left\{\left(h_{1}, .3\right),\left(h_{2}, .4\right),\left(h_{3}, .5\right)\right\}$

(ii) $(\widetilde{F}, A) \widetilde{\subseteq}(\widetilde{G}, B)$

\section{Fuzzy soft matrices}

Let $U=\left\{u_{1}, u_{2}, \ldots, u_{m}\right\}$ be the universal set and $E$ be the set of parameters given by $E=\left\{e_{1}, e_{2}, \ldots, e_{n}\right\}$. Then the fuzzy soft set $(\widetilde{F}, E)$ can be expressed in matrix form as $\widetilde{A}=\left[a_{i j}\right]_{m \times n}$ or simply by $\left[a_{i j}\right], i=1,2, \ldots, m, j=1,2, \ldots, n$ and $\left[a_{i j}\right]=\left[\left(\mu_{i j}, \gamma_{i j}\right)\right]$; where $\mu_{i j}$ and $\gamma_{i j}$ represent the fuzzy membership function and fuzzy reference function respectively of $u_{i}$ in the fuzzy set $\widetilde{F}\left(e_{j}\right)$ so that $\delta_{i j}=\mu_{i j}-\gamma_{i j}$ gives the fuzzy membership value of $u_{i}$. We shall identify a fuzzy soft set with its fuzzy soft matrix and use these two concepts interchangeable. The set of all $m \times n$ fuzzy soft matrices over $U$ will be denoted by $F S M_{m \times n}$. For usual fuzzy sets with fuzzy reference function 0 , it is obvious to see that $\left[a_{i j}\right]=\left[\left(\mu_{i j}, 0\right)\right]$ for all $i, j$.

Example 2. Let $U=\left\{u_{1}, u_{2}, u_{3}\right\}$ be the universal set and $E$ be the set of parameters given by $E=\left\{e_{1}, e_{2}, e_{3}\right\}$. We consider a fuzzy soft set

$$
\begin{gathered}
(\widetilde{F}, E)=\left\{\widetilde{F}\left(e_{1}\right)=\left\{\left(U_{1}, 0.3,0\right),\left(U_{2}, 0.4,0\right),\left(U_{3}, 0.7,0\right)\right\},\right. \\
\widetilde{F}\left(e_{2}\right)=\left\{\left(U_{1}, 0.9,0\right),\left(U_{2}, 0.2,0\right),\left(U_{3}, 0.4,0\right)\right\}, \\
\left.\widetilde{F}\left(e_{3}\right)=\left\{\left(U_{1}, 0.8,0\right),\left(U_{2}, 0.1,0\right),\left(U_{3}, 0.3,0\right)\right\}\right\} .
\end{gathered}
$$

We would represent this fuzzy soft set in matrix form as

$$
\left[a_{i j}\right]_{3 \times 3}=\left[\begin{array}{ccc}
(0.3,0) & (0.9,0) & (0.8,0) \\
(0.4,0) & (0.2,0) & (0.1,0) \\
(0.7,0) & (0.4,0) & (0.3,0)
\end{array}\right]_{3 \times 3} .
$$

Definition 8. The membership value matrix corresponding to the matrix $\widetilde{A}$ as $M V(\widetilde{A})=\left[\delta_{i j}\right]_{m \times n}$, where $\delta_{\tilde{i j}}=\mu_{\tilde{i j}}-\gamma_{i j}$ $\forall i=1,2, \ldots, m$ and $j=1,2, \ldots, n . \mu_{i j}$ and $\gamma_{i j}$ represent the fuzzy membership function and fuzzy reference function respectively of $u_{i}$ in the fuzzy set $\widetilde{F}\left(e_{j}\right)$. 
Definition 9. Let $\widetilde{A}=\left[a_{i j}\right] \in F S M_{m \times n}$ where $a_{i j}=\left(\mu_{i j}, \gamma_{i j}\right)$. Then

(i) $\widetilde{A}$ is called a null fuzzy soft matrix denoted by $[\widetilde{0}]_{m \times n}$ or simply by $[\widetilde{0}]$ if $\delta_{i j}=\widetilde{0}$ for all $i$ and $j$. For usual fuzzy sets, $\delta_{i j}=\mu_{i j} \forall i, j$.

(ii) $\widetilde{A}$ is called a identify fuzzy soft matrix denoted by $[\widetilde{1}]$, if $m=n, a_{i j}=\left(\mu_{i j}, \gamma_{i j}\right)$ for all $i \neq j$ and $a_{i j}=(1,0)$ i.e, $\delta_{i j}=1$ for all $i=j$.

Definition 10. Let $\widetilde{A}=\left[\left(a_{i j}, 0\right)\right]_{\sim c} \in F S M_{m \times n}$, where $a_{i j}=\left(\mu_{i j}, \gamma_{i j}\right)$. Then the representation of the complement of the fuzzy matrix $\widetilde{A}$ which is denoted by $\tilde{\sim}^{c}$ and then $\tilde{\sim}^{c}$ is called fuzzy soft complement matrix if $\tilde{A}^{c}=\left[\left(1, a_{i j}\right)\right]_{m \times n}$ for all $a_{i j} \in[0,1]$. Then the matrix obtained from so called membership value would be the following $\stackrel{\sim}{A}=\left[a_{i j}\right]=\left[\left(1-a_{i j}\right)\right]$ for all $i, j$.

Definition 11. Let $\widetilde{A}=\left[a_{i j}\right]_{m \times n}, a_{i j}=\left(\mu_{i j}, \gamma_{i j}\right)$, where $\mu_{i j}$ and $\gamma_{i j}$ represent the fuzzy membership function and fuzzy reference function respectively of $u_{i}$. Also let $\widetilde{B}=\left[b_{j k}\right]_{n \times p}, b_{j k}=\left(\mu_{j k}, \gamma_{j k}\right)$, where $\mu_{j k}$ and $\gamma_{j k}$ represent the fuzzy membership function and fuzzy reference function respectively of $u_{i} . \delta_{j k}=\mu_{j k}-\gamma_{j k}$ gives the fuzzy membership value of $u_{i}$. We now define $\widetilde{A} \cdot \widetilde{B}$, the product of $\widetilde{A}$ and $\widetilde{B}$ as $\widetilde{A} \cdot \widetilde{B}=\left[d_{i k}\right]_{m \times p}=\left[\max \min \left(\mu_{i j}, \mu_{j k}\right) \text {, } \min \max \left(\gamma_{i j}, \gamma_{j k}\right)\right]_{m \times p}, 1 \leq i \leq m$, $1 \leq k \leq$ for all $j=1,2, . ., n$.

\section{Methodology and algorithm}

In this section we present an algorithm for medical diagnosis using fuzzy soft matrices. Assume that there is a set of $m$ patients, $P=\left\{p_{1}, p_{2}, p_{3}, \ldots . ., p_{m}\right\}$ with a set of $n$ symptoms $S=\left\{s_{1}, s_{2}, s_{3}, \ldots . . s_{n}\right\}$ related to a set of $k$ diseases $D=\left\{d_{1}, d_{2}, d_{3}, \ldots, d_{k}\right\}$.

We apply fuzzy soft set theory to develop a technique through Sanchez's method to diagnose which patient is suffering from what disease by using fuzzy soft matrices.

For this, construct a fuzzy soft set $(\widetilde{F}, D)$ over $S$ where $\widetilde{F}$ is a mapping $\widetilde{F}: D \rightarrow \mathscr{F}(S)$. This fuzzy soft set gives a relation matrix $\widetilde{A}$, called symptom-disease matrix, where each element denote the weight of the symptoms for a certain disease.Also, its complement $(\widetilde{F}, D)^{c}$ gives another relation matrix $\widetilde{\sim}^{c}$ called non symptom-disease matrix. We call the matrices $\widetilde{A}$ and $\tilde{A}^{c}$ as medical knowledge of fuzzy soft set.

Then construct another fuzzy soft set $(\widetilde{G}, S)$ over $P$, where $\widetilde{G}$ is a mapping $\widetilde{G}: S \rightarrow \mathscr{F}(P)$. This fuzzy soft set gives a relation matrix $\widetilde{B}$, called patient-symptom matrix. Similarly, its complement $(\widetilde{G}, S)^{c}$ gives the relation matrix $\widetilde{B}^{c}$ called patient-non symptom matrix.

Thus the general form of $\widetilde{A}$ and $\tilde{A}^{c}$ are

$$
\widetilde{A}=\begin{gathered}
d_{1} \\
s_{1} \\
s_{2} \\
\cdot \\
\cdot \\
s_{n}
\end{gathered}\left[\begin{array}{ccccc}
a_{11} & a_{12} & a_{13} & \ldots & d_{1 n} \\
a_{21} & a_{22} & a_{23} & \ldots & a_{2 n} \\
a_{31} & a_{32} & a_{33} & \ldots & a_{3 n} \\
\cdot & . & . & \ldots & . \\
\cdot & . & . & \ldots & . \\
\cdot & . & . & \ldots & . \\
a_{m 1} & a_{m 2} & a_{m 3} & \ldots & a_{n k}
\end{array}\right]
$$




$$
\begin{aligned}
& \begin{array}{lllll}
d_{1} & d_{2} & d_{3} & \ldots & d_{k}
\end{array}
\end{aligned}
$$

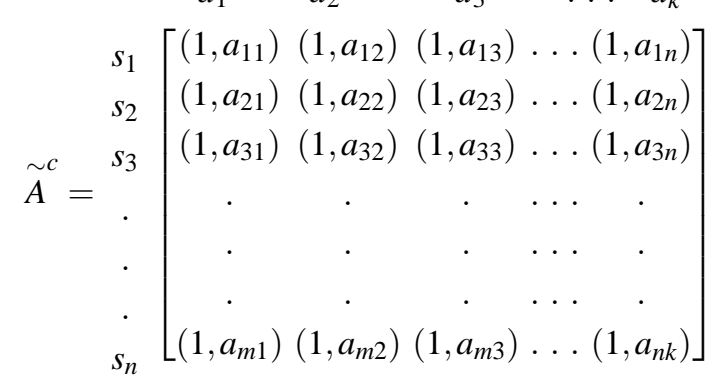

and the general form of $\widetilde{B}$ and $\widetilde{B}^{c}$ are

$$
\begin{aligned}
& \widetilde{B}=\begin{array}{c}
p_{1} \\
p_{2} \\
p_{3} \\
\cdot \\
\cdot \\
\cdot \\
p_{m}
\end{array}\left[\begin{array}{ccccc}
s_{11} & s_{2} & s_{3} & \ldots & s_{n} \\
b_{21} & b_{12} & b_{13} & \ldots & b_{1 k} \\
b_{31} & b_{32} & b_{33} & \ldots & b_{3 k} \\
\cdot & \cdot & \cdot & \ldots & \cdot \\
\cdot & \cdot & \cdot & \ldots & \cdot \\
\cdot & \cdot & \cdot & \ldots & \cdot \\
b_{n 1} & b_{n 2} & b_{n 3} & \ldots & b_{m n}
\end{array}\right]
\end{aligned}
$$

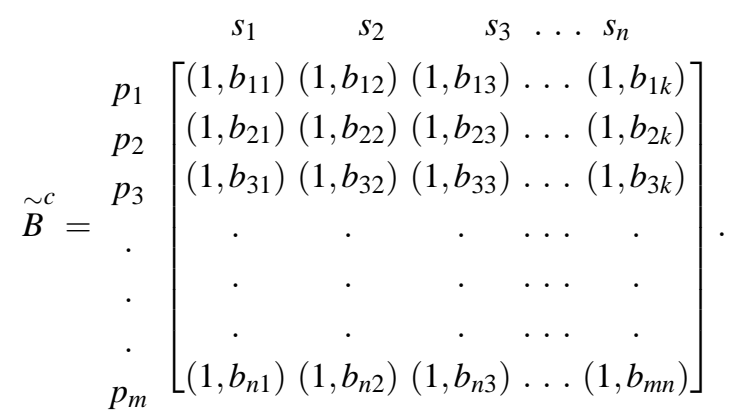

Then using Definition (11), we obtain two new relation matrices $\widetilde{T}_{1}=\widetilde{B} \cdot \widetilde{A}$ and $\widetilde{T_{2}}=\widetilde{B} \cdot \tilde{A}^{c}$ called patient symptom disease matrix and patient symptom non disease matrix respectively. In similarly, we obtain the relation matrices $\widetilde{T}_{3}=\stackrel{\sim}{B}^{c} \cdot \widetilde{A}$ and $\widetilde{T}_{4}=\stackrel{\sim_{B}^{c}}{\sim_{A}^{c}}$ called the patient non symptom disease matrix and patient non symptom non disease matrix respectively.

Using Definition (8), we may obtain the corresponding membership value matrices $M V\left(\widetilde{T}_{1}\right), M V\left(\widetilde{T}_{2}\right), M V\left(\widetilde{T}_{3}\right)$ and $M V\left(\widetilde{T}_{4}\right)$. We calculate the diagnosis score $S_{\widetilde{T}_{1}}$ and $S_{\widetilde{T_{2}}}$ for and against the disease respectively as

$$
S_{\widetilde{T}_{1}}=\left[\gamma\left(\widetilde{T}_{1}\right)_{i j}\right]_{m \times n}, \text { where } \gamma\left(\widetilde{T}_{1}\right)_{i j}=\delta\left(\widetilde{T}_{1}\right)_{i j}-\delta\left(\widetilde{T}_{3}\right)_{i j}
$$

and

$$
S_{\widetilde{T_{2}}}=\left[\gamma\left(\widetilde{T}_{2}\right)_{i j}\right]_{m \times n}, \text { where } \gamma\left(\widetilde{T}_{2}\right)_{i j}=\delta\left(\widetilde{T}_{2}\right)_{i j}-\delta\left(\widetilde{T}_{4}\right)_{i j} .
$$

Now if $\max _{j}\left[S_{\widetilde{T}_{1}}\left(p_{i}, d_{j}\right)-S_{\widetilde{T}_{2}}\left(p_{i}, d_{j}\right)\right]$ occurs for exactly $\left(p_{i}, d_{k}\right)$ only, then we would be in a position to accept that diagnostic hypothesis for patient $p_{i}$ is the disease $d_{k}$. In case there is a tie, the process is repeated for patient $p_{i}$ by reassessing the symptoms. 


\subsection{Algorithm}

Step I: Input the fuzzy soft set $(\widetilde{F}, D)$ and compute $(\widetilde{F}, D)^{c}$. Compute the corresponding matrices $\widetilde{A}$ and $\widetilde{A}^{c}$. Step II: Input the fuzzy soft set $(\widetilde{G}, S)$ and compute $(\widetilde{G}, S)^{c}$. Compute the corresponding matrices $\widetilde{B}$ and $\widetilde{\sim}^{c}$. Step III: Compute $\widetilde{T}_{1}=\widetilde{B} \cdot \widetilde{A}, \widetilde{T}_{2}=\widetilde{B} \cdot \widetilde{A}, \widetilde{T_{3}}=\stackrel{\sim}{B} \cdot \widetilde{A}$ and $\widetilde{T}_{4}=\stackrel{\sim}{B} \cdot \widetilde{\sim}^{c}$.

Step $I V$ : Compute the corresponding membership value matrices $M V\left(\widetilde{T}_{1}\right), M V\left(\widetilde{T}_{2}\right), M V\left(\widetilde{T}_{3}\right)$ and $M V\left(\widetilde{T}_{4}\right)$.

Step V: Compute the diagnosis score $S_{\widetilde{T}_{1}}$ and $S_{\widetilde{T}_{2}}$.

Step VI: Find $S_{k}=\max _{j}\left[S_{\widetilde{T}_{1}}\left(p_{i}, d_{j}\right)-S_{\widetilde{T}_{2}}\left(p_{i}, d_{j}\right)\right]$.

Then we conclude that the patient $p_{i}$ is suffering from the disease $d_{k}$. If $S_{k}$ has more than one value, then go to step I and repeat the process by reassessing the symptoms for the patient.

\section{Case study}

Suppose there are three patients John, George and Albert in a hospital those who intake over dosage for sensual pleasure which will affect the brain cells lead to the symptoms of hysteria, then the patient who used sleeping pills will have the side affect of headache and stomach pain, then the patient who take birth control pills will have side effect of depression and stroke. We consider the set $S=\left\{s_{1}, s_{2}, s_{3}\right\}$ as universal set where $s_{1}, s_{2}, s_{3}$ represent symptoms of hysteria, headache and stomach pain, depression and stroke problems respectively and the set $D=\left\{d_{1}, d_{2}\right\}$ where $d_{1}$ and $d_{2}$ represent the parameters of side effect in the human body, particularly brain and heart problem disease respectively.

Suppose,

$$
\begin{aligned}
(\widetilde{F}, D) & =\left\{\widetilde{F}\left(d_{1}\right)=\left\{\left(s_{1}, 0.4,0\right),\left(s_{2}, 0.7,0\right),\left(s_{3}, 0.6,0\right)\right\}\right. \\
\widetilde{F}\left(d_{2}\right) & \left.=\left\{\left(s_{1}, 0.9,0\right),\left(s_{2}, 0.8,0\right),\left(s_{3}, 0.5,0\right)\right\}\right\}
\end{aligned}
$$

Complement of $(\widetilde{F}, D)$ is given by

$$
\begin{gathered}
(\widetilde{F}, D)^{c}=\left\{\widetilde{F}^{c}\left(d_{1}\right)=\left\{\left(s_{1}, 1,0.4\right),\left(s_{2}, 1,0.7\right),\left(s_{3}, 1,0.6\right)\right\}\right. \\
\left.\widetilde{F}^{c}\left(d_{2}\right)=\left\{\left(s_{1}, 1,0.9\right),\left(s_{2}, 1,0.8\right),\left(s_{3}, 1,0.5\right)\right\}\right\}
\end{gathered}
$$

We represent the fuzzy soft set $(\widetilde{F}, D)$ and $(\widetilde{F}, D)^{c}$ by the following matrices $\widetilde{A}$ and $\tilde{A}^{c}$ respectively.

$$
\begin{aligned}
& d_{1} \quad d_{2} \\
& \widetilde{A}=\begin{array}{l}
p_{1} \\
p_{2} \\
p_{3}
\end{array}\left[\begin{array}{ll}
(0.4,0) & (0.9,0) \\
(0.7,0) & (0.8,0) \\
(0.6,0) & (0.5,0)
\end{array}\right] \\
& d_{1} \quad d_{2} \\
& \tilde{A}^{c}=\begin{array}{l}
p_{1} \\
p_{2} \\
p_{3}
\end{array}\left[\begin{array}{ll}
(1,0.4) & (1,0.9) \\
(1,0.7) & (1,0.8) \\
(1,0.6) & (1,0.5)
\end{array}\right] .
\end{aligned}
$$


Next suppose,

$$
\begin{gathered}
(\widetilde{G}, S)=\left\{\widetilde{G}\left(s_{1}\right)=\left\{\left(p_{1}, 0.6,0\right),\left(p_{2}, 0.8,0\right),\left(p_{3}, 0.2,0\right)\right\}\right. \\
\widetilde{G}\left(s_{2}\right)=\left\{\left(p_{1}, 0.9,0\right),\left(p_{2}, 0.5,0\right),\left(p_{3}, 0.4,0\right)\right\} \\
\left.\widetilde{G}\left(s_{3}\right)=\left\{\left(p_{1}, 0.3,0\right),\left(p_{2}, 0.9,0\right),\left(p_{3}, 0.8,0\right)\right\}\right\} .
\end{gathered}
$$

Complement of $(\widetilde{G}, S)$ is given by

$$
\begin{gathered}
(\widetilde{G}, S)^{c}=\left\{\widetilde{G}^{c}\left(s_{1}\right)=\left\{\left(p_{1}, 1,0.6\right),\left(p_{2}, 1,0.8\right),\left(p_{3}, 1,0.2\right)\right\},\right. \\
\widetilde{G}^{c}\left(s_{2}\right)=\left\{\left(p_{1}, 1,0.9\right),\left(p_{2}, 1,0.5\right),\left(p_{3}, 1,0.4\right)\right\} \\
\left.\widetilde{G}^{c}\left(s_{3}\right)=\left\{\left(p_{1}, 1,0.3\right),\left(p_{2}, 1,0.9\right),\left(p_{3}, 1,0.8\right)\right\}\right\} .
\end{gathered}
$$

We represent the fuzzy soft set $(\widetilde{G}, S)$ and $(\widetilde{G}, S)^{c}$ by the following matrices $\widetilde{B}$ and $\stackrel{\sim^{c}}{B}$ respectively.

$$
\begin{aligned}
& \begin{array}{lll}
s_{1} & s_{2} & s_{3}
\end{array} \\
& \widetilde{B}=\begin{array}{l}
p_{1} \\
p_{2} \\
p_{3}
\end{array}\left[\begin{array}{lll}
(0.6,0) & (0.8,0) & (0.2,0) \\
(0.9,0) & (0.5,0) & (0.4,0) \\
(0.3,0) & (0.9,0) & (0.8,0)
\end{array}\right] \\
& \tilde{\sim}^{c}=\begin{array}{l}
p_{1} \\
p_{2} \\
p_{3}
\end{array}\left[\begin{array}{lll}
(1,0.6) & (1,0.8) & (1,0.2) \\
(1,0.9) & (1,0.5) & (1,0.4) \\
(1,0.3) & (1,0.9) & (1,0.8)
\end{array}\right]
\end{aligned}
$$

Thus we have

$$
\begin{aligned}
& d_{1} \quad d_{2} \\
& \widetilde{T_{1}}=\widetilde{B} \cdot \widetilde{A}=\begin{array}{c}
s_{1} \\
s_{2} \\
s_{3}
\end{array}\left[\begin{array}{ll}
(0.7,0) & (0.8,0) \\
(0.5,0) & (0.9,0) \\
(0.7,0) & (0.8,0)
\end{array}\right] \\
& d_{1} \quad d_{2} \\
& \widetilde{T_{2}}=\widetilde{B} \cdot \tilde{A}^{c}=\begin{array}{l}
s_{1} \\
s_{2} \\
s_{3}
\end{array}\left[\begin{array}{ll}
(0.8,0.4) & (0.8,0.5) \\
(0.9,0.4) & (0.9,0.5) \\
(0.9,0.4) & (0.9,0.4)
\end{array}\right] \\
& d_{1} \quad d_{2} \\
& \widetilde{T}_{3}=\stackrel{\sim}{B} . \widetilde{A}=\begin{array}{l}
p_{1} \\
p_{2} \\
p_{3}
\end{array}\left[\begin{array}{ll}
(0.7,0.2) & (0.9,0.2) \\
(0.7,0.4) & (0.9,0.4) \\
(0.7,0.3) & (0.9,0.3)
\end{array}\right] \\
& d_{1} \quad d_{2}
\end{aligned}
$$

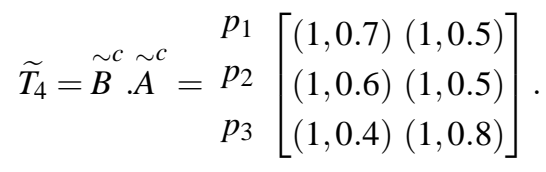


Then we have following membership value matrices $M V\left(\widetilde{T}_{1}\right), M V\left(\widetilde{T}_{2}\right), M V\left(\widetilde{T}_{3}\right)$ and $M V\left(\widetilde{T}_{4}\right)$

$$
\begin{aligned}
& d_{1} \quad d_{2} \\
& M V\left(\widetilde{T}_{1}\right)=\begin{array}{l}
p_{1} \\
p_{2} \\
p_{3}
\end{array}\left[\begin{array}{ll}
0.7 & 0.8 \\
0.5 & 0.9 \\
0.7 & 0.8
\end{array}\right] \\
& d_{1} \quad d_{2} \\
& M V\left(\widetilde{T}_{2}\right)=\begin{array}{l}
p_{1} \\
p_{2} \\
p_{3}
\end{array}\left[\begin{array}{ll}
0.4 & 0.3 \\
0.5 & 0.4 \\
0.5 & 0.5
\end{array}\right] \\
& d_{1} \quad d_{2} \\
& M V\left(\widetilde{T}_{3}\right)=\begin{array}{l}
p_{1} \\
p_{2} \\
p_{3}
\end{array}\left[\begin{array}{ll}
0.5 & 0.7 \\
0.3 & 0.5 \\
0.4 & 0.6
\end{array}\right] \\
& d_{1} \quad d_{2} \\
& M V\left(\widetilde{T}_{4}\right)=\begin{array}{l}
p_{1} \\
p_{2} \\
p_{3}
\end{array}\left[\begin{array}{ll}
0.3 & 0.5 \\
0.4 & 0.5 \\
0.6 & 0.2
\end{array}\right] .
\end{aligned}
$$

We conclude the diagnosis score $S_{\widetilde{T}_{1}}$ and $S_{\widetilde{T}_{2}}$ for and against the disease as below.

$$
\begin{aligned}
& d_{1} \quad d_{2} \\
& S_{\widetilde{T}_{1}}=\begin{array}{l}
p_{1} \\
p_{2} \\
p_{3}
\end{array}\left[\begin{array}{ll}
0.2 & 0.1 \\
0.2 & 0.4 \\
0.3 & 0.2
\end{array}\right] \\
& d_{1} \quad d_{2} \\
& S_{\widetilde{T_{2}}}=\begin{array}{l}
p_{1} \\
p_{2} \\
p_{3}
\end{array}\left[\begin{array}{cc}
0.1 & -0.2 \\
0.1 & -0.1 \\
-0.1 & 0.3
\end{array}\right] .
\end{aligned}
$$

Now we have the difference for and against the disease

\begin{tabular}{|l|l|l|}
\hline$S_{\widetilde{T}_{1}}-S_{\widetilde{T}_{2}}$ & $d_{1}$ & $d_{2}$ \\
\hline$p_{1}$ & 0.1 & 0.3 \\
\hline$p_{2}$ & 0.1 & 0.5 \\
\hline$p_{3}$ & 0.4 & -0.1 \\
\hline
\end{tabular}

It is clear from the above matrix that patients $p_{1}$ and $p_{2}$ are suffering from disease $d_{2}$, patient $p_{3}$ is suffering from disease $d_{1}$.

\section{Conclusions}

We have applied the notion of fuzzy soft matrices in the field of medical diagnosis. A case study has been taken to exhibit the simplicity of the technique. Future work in this regard would be required to study whether the notions put forward in this paper yield a fruitful result. 


\section{References}

[1] L.A. Zadeh, Fuzzy sets, Inf. Control, 8, 338-353, (1965).

[2] Z. Pawlak, Rough sets, Int. J. Inf. Comp. Sci., 11, 341-356, (1982).

[3] D. Molodtsov, Soft set theory-first result, Computers and Mathematics with Applications, 37, 19-31, (1999).

[4] P.K. Maji, R. Biswas and A.R. Roy, Fuzzy Soft Set, The Journal of Fuzzy Mathematics, 9(3), 677-692, (2001).

[5] T.J. Neog and D.K. Sut, Theory of Fuzzy Soft Sets From a New Perspective, International Journal of Latest Trends in Computing, 2(3), 439-450, (2011).

[6] S.K. De, R. Biswas and A.R. Roy, An Application of Intuitionistic fuzzy sets in medical diagnosis, Fuzzy Sets and Systems, 117, 209-213, (2001).

[7] E. Sanchez, Resolution of composite fuzzy relation equations, Information and control, 30, 38-48, (1976).

[8] E. Sanchez, Inverse of fuzzy relations, Application to possibility distributions and medical diagnosis, Fuzzy Sets and Systems, 2(1), 75-86, (1979).

[9] B.K. Saikia, P.K. Das and A.K. Borkakati, An Application of Intuitionistic fuzzy soft sets in medical diagnosis, Bio Science Research Bulletin, 19(2), 121-127, (2003).

[10] B. Chetia and P.K. Das, An Application of Interval valued fuzzy soft set in medical diagnosis, Int.J.contempt. math. sciences, 5(38), 1887-1894,(2010).

[11] A.R. Meenakshi and M. Kaliraja, An Application of Interval Valued Fuzzy Matrices in Medical Diagnosis, Int. Journal of Math. Analysis, 5(36), 1791-1802, (2011).

[12] MI. Ali and M. Shabir Comments on De Morgan's law in fuzzy soft sets. J. Fuzzy Math., 18(3), 679-686, (2010). 